\title{
Spectrum of the Intensity of Modulated Noisy Light After Propagation in Dispersive Fiber
}

\author{
W. K. Marshall and A. Yariv, Life Fellow, IEEE
}

\begin{abstract}
The spectral density of the optical intensity which results after modulated, noisy light is propagated in dispersive single-mode fiber is investigated theoretically and experimentally. An exact general result is obtained for the case of lowest-order-only group velocity dispersion and is applied to light from a $1550-\mathrm{nm}$ distributed-feedback semiconductor laser which is large-signal phase modulated and then propagated through $50 \mathrm{~km}$ of standard single-mode fiber. Experimental results demonstrate the effect of dispersion on the intensity spectrum (and thus, on lightwave system characteristics such as modulation response, relative intensity noise, carrier-to-noise ratio, and harmonic distortion) in this situation and provide confirmation of the theoretical results.
\end{abstract}

Index Terms-Laser noise, optical fiber communications, optical propagation in dispersive media, spectral analysis.

D ISPERSIVE propagation has long been known as a major factor limiting the performance of lightwave transmission at $1550 \mathrm{~nm}$. Although the effect of group velocity dispersion (GVD) on the optical field is relatively easy to understand, the effect on optical intensity (and hence, following direct detection, the received electrical signal) can be more difficult to determine. Reasons for this include 1) the field spectrum input to the fiber in state-of-the-art systems may be quite complicated, resulting from combinations of amplitude and phase variations, some of which may have large amplitudes; 2) field amplitude and phase variations are interconverted by dispersive propagation; and 3) the output intensity is related to the output field amplitude by a square-law, so that lightwave transmission is nonlinear even in cases where the underlying field transmission is linear. These factors limit intuitive understanding of the lightwave channel and can lead to difficulty in predicting the dependence of system characteristics such as modulation response, relative intensity noise, and harmonic distortion on propagation distance, modulation format, and source laser characteristics.

A number of authors [1]-[7] have considered the effects of dispersion on modulated pure carriers (without noise) or on noisy light without modulation. The influence of intensity modulation on the intensity noise which results, after propagation, from phase noise in a semiconductor laser was considered in [8] and [9], but only in the limit of weak dispersion (and thus, narrow system bandwidth and/or small propagation distance). In [10], we reported an exact general formula for the spectral

\footnotetext{
Manuscript received October 1, 1999; revised December 2, 1999. This work was supported by the Office of Naval Research, the Defense Advanced Research Project Agency, and the Air Force Office of Scientific Research under Grant N00014-91-J-1195.

The authors are with the Department of Applied Physics, California Institute of Technology, Pasadena, CA 91125 USA.

Publisher Item Identifier S 1041-1135(00)01994-7.
}

density of the intensity of ergodic (hence, unmodulated) light after propagation in the case of lowest order-only GVD.

In this paper, we extend our general formula so as to hold for arbitrary, nonergodic input fields, including modulated, noisy ones. We then apply this formula to a situation involving a 1550-nm distributed-feedback (DFB) semiconductor laser followed by large-index phase modulation at 4 or $12.8 \mathrm{GHz}$. We thus model, and then measure, the microwave $(1-25 \mathrm{GHz})$ intensity spectrum which results from this input after propagation through $50 \mathrm{~km}$ of standard single-mode fiber (SMF). Without fiber, the intensity spectrum is unaffected by the phase modulation. With fiber, the intensity spectrum is affected in agreement with the results of our theory. Previously described theoretical approaches are difficult or impossible to use in understanding these experimental results.

Linear propagation with lowest order-only group velocity dispersion is described by the field envelope equation

$$
\frac{\partial \mathcal{E}}{\partial z}+\beta_{0}^{\prime} \frac{\partial \mathcal{E}}{\partial t}-\frac{i}{2} \beta_{0}^{\prime \prime} \frac{\partial^{2} \mathcal{E}}{\partial t^{2}}+\frac{\alpha}{2} \mathcal{E}=0
$$

where $\beta_{0}^{\prime}$ is the group delay per unit length; $\beta_{0}^{\prime \prime}$ is the group delay dispersion per unit angular frequency per unit length; and $\alpha$ is the fiber loss per unit distance. After absorbing the group delay $\beta_{0}^{\prime} z$ into the time variable $t$, (1) leads to

$$
\mathcal{E}(t, z)=e^{-(\alpha / 2) z} \int_{-\infty}^{\infty} \mathcal{E}_{0}(t+s) \frac{e^{\left(i s^{2} / 2 \beta_{0}^{\prime \prime} z\right)} e^{ \pm(i \pi / 4)}}{\sqrt{2 \pi\left|\beta_{0}^{\prime \prime} z\right|}} d s
$$

where $\mathcal{E}_{0}(t)$ is the envelope of the electric field at $z=0$. The Wiener-Khintchine theorem defines the spectral density of the intensity $S_{\mathrm{II}}(\Omega, z)$ as

$$
S_{\mathrm{II}}(\Omega, z)=2 \int_{-\infty}^{\infty} R_{\mathrm{II}}(\tau, z) e^{-i \Omega \tau} d \tau
$$

where

$$
R_{\mathrm{II}}(\tau, z) \equiv \lim _{T \rightarrow \infty} \frac{1}{2 T} \int_{-T}^{T} I(z, t) I(z, t+\tau) d t
$$

is the autocorrelation of the intensity at $z$.

The above equations lead, after significant algebra (see [10]), but without any approximation, to our fundamental result for the spectral density of the intensity after propagation with lowest order-only GVD

$$
S_{\mathrm{II}}(\Omega, z)=2 e^{-\alpha z} \int_{-\infty}^{\infty} R_{4}\left(\beta_{0}^{\prime \prime} z \Omega, \beta_{0}^{\prime \prime} z \Omega+u, u\right) e^{-i \Omega u} d u
$$


where $R_{4}$ is the fourth-order input field envelope correlation

$$
\begin{aligned}
R_{4}\left(\tau_{1}, \tau_{2}, \tau_{3}\right) \equiv \lim _{T \rightarrow \infty} \frac{1}{2 T} \int_{-T}^{T} \mathcal{E}_{0}^{*}(t) \mathcal{E}_{0}\left(t+\tau_{1}\right) \\
\mathcal{E}_{0}^{*}\left(t+\tau_{2}\right) \mathcal{E}_{0}\left(t+\tau_{3}\right) d t .
\end{aligned}
$$

A corresponding result for the relative intensity spectrum, which is the spectrum of normalized intensity variation, $\{I(z, t)-\bar{I}(z)\} / \bar{I}(z)$, where $\bar{I}(z)$ is the time-average intensity at $z$, is given by the right-hand side of (3) with $R_{\mathrm{II}}(\tau, z)$ replaced by $\left\{R_{\mathrm{II}}(\tau, z) / \bar{I}^{2}(z)\right\}-1$. Thus,

$\operatorname{RIN}(\Omega, z)=2 \int_{-\infty}^{\infty}\left\{\frac{R_{4}\left(\beta_{0}^{\prime \prime} z \Omega, \beta_{0}^{\prime \prime} z \Omega+u, u\right)}{R_{2}^{2}(0)}-1\right\} e^{-i \Omega u} d u$

where $R_{2}$ is the second-order input field envelope correlation function

$$
R_{2}(\tau) \equiv \lim _{T \rightarrow \infty} \frac{1}{2 T} \int_{-T}^{T} \mathcal{E}_{0}^{*}(t) \mathcal{E}_{0}(t+\tau) d t
$$

and it has been used that $\bar{I}(z) \equiv e^{-\alpha z} R_{2}(0)$.

When all of the variations in the intensity are due to intensity noise, $\operatorname{RIN}(\Omega, z)$ is the (usual) relative intensity noise (RIN) factor. When variations include a modulation tone or subcarrier, the resulting "RIN" contains delta functions due to the modulation and gives, therefore, information about the carrier-to-noise ratio (CNR). When variations include those due to an out-of-band modulation, or "dither," the resulting spectrum gives information about the RIN expected under operating conditions which include the dither. In the latter cases, a considerable simplification in the use of the above formulas is obtained by introducing an input field envelope of the form $\mathcal{E}_{0}(t)=\mathcal{E}_{0}^{(a)}(t) \mathcal{E}_{0}^{(b)}(t)$. Then, as long as variations in the envelopes $(a)$ and $(b)$ are uncorrelated, ${ }^{1}$ we will have

$$
\begin{gathered}
R_{2}(\tau)=R_{2}^{(a)}(\tau) R_{2}^{(b)}(\tau) \\
R_{4}\left(\tau_{1}, \tau_{2}, \tau_{3}\right)=R_{4}^{(a)}\left(\tau_{1}, \tau_{2}, \tau_{3}\right) R_{4}^{(b)}\left(\tau_{1}, \tau_{2}, \tau_{3}\right)
\end{gathered}
$$

where $R_{2}^{(a)}$ is defined by (8) with $\mathcal{E}_{0}$ replaced by $\mathcal{E}_{0}^{(a)}$, etc. These formulas can be extended to cases of multiple sequential modulations by further taking $\mathcal{E}_{0}^{(b)}(t)=\mathcal{E}_{0}^{(c)}(t) \mathcal{E}_{0}^{(d)}(t)$, etc. All of the above results apply both to deterministic (modulated pure carrier) and to stochastic (noisy, possibly modulated) input fields. Their usefulness exceeds the scope of the application made in the remainder of this letter.

For a pure carrier with sinusoidal phase modulation at angular frequency $\Omega_{1}$ and modulation index $\beta_{\mathrm{FM}}$ [i.e., $\mathcal{E}_{0}^{(a)}(t)=1$ and $\left.\mathcal{E}_{0}^{(b)}(t)=e^{i \beta_{\mathrm{FM}} \cos \Omega_{1} t}\right]$, (6) leads to

$R_{4}^{(b)}\left(\beta_{0}^{\prime \prime} z \Omega, \beta_{0}^{\prime \prime} z \Omega+u, u\right)=\sum_{k=-\infty}^{\infty} J_{k}^{2}\left(2 \beta_{\mathrm{FM}} \sin \frac{\beta_{0}^{\prime \prime} z \Omega \Omega_{1}}{2}\right) e^{i k \Omega_{1} u}$

where it has been used that $\cos a-\cos b=-2 \sin ((a-$ b)/2) $\sin ((a+b) / 2)$, that $e^{-i x \sin y}=\sum_{p=-\infty}^{\infty} J_{p}(x) e^{-i p y}$,

${ }^{1}$ Specific requirements for (9) and (10) to hold are that $\Delta_{2}^{(a)}$ is uncorrelated with $\Delta_{2}^{(b)}$ and $\Delta_{4}^{(a)}$ is uncorrelated with $\Delta_{4}^{(b)}$, where $\Delta_{2}^{(a)} \equiv \mathcal{E}_{0}^{(a)}(t)^{*} \mathcal{E}_{0}^{(a)}(t+\tau)-R_{2}^{(a)}(\tau), \Delta_{4}^{(a)} \equiv \mathcal{E}_{0}^{(a)}(t)^{*} \mathcal{E}_{0}^{(a)}(t+$ $\left.\tau_{1}\right) \mathcal{E}_{0}^{(a)}\left(t+\tau_{2}\right)^{*} \mathcal{E}_{0}^{(a)}\left(t+\tau_{3}\right)-R_{4}^{(a)}\left(\tau_{1}, \tau_{2}, \tau_{3}\right)$, and $\Delta_{2}^{(b)}$ and $\Delta_{4}^{(b)}$ are defined similarly but with (b) replacing (a).

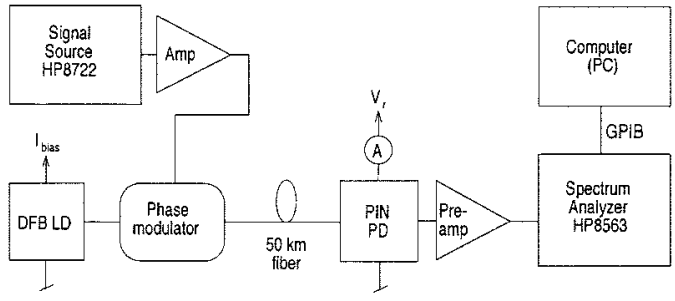

Fig. 1. Experimental setup.

and that $\lim _{T \rightarrow \infty}(1 / 2 T) \int_{-T}^{T} e^{-i(p+q) \Omega_{1} t} d t=1$ if $q=-p$ and zero otherwise. Using (7) and noting that (8) gives $R_{2}^{(a)}(0)=R_{2}^{(b)}(0)=1$ in this case, we obtain

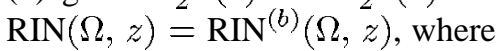

$\operatorname{RIN}^{(b)}(\Omega, z)=8 \pi \sum_{k=1}^{\infty} J_{k}^{2}\left(2 \beta_{\mathrm{FM}} \sin \frac{k \beta_{0}^{\prime \prime} z \Omega_{1}^{2}}{2}\right) \delta\left(\Omega-k \Omega_{1}\right)$.

The "RIN" in this case is just a sum of delta functions at $\Omega=$ $k \Omega_{1}$, for $k \geq 1$, representing the modulation.

For a noisy carrier with sinusoidal phase modulation [where $\mathcal{E}_{0}^{(b)}(t)=e^{i \beta_{\mathrm{FM}} \cos \Omega_{1} t}$ and $\mathcal{E}_{0}^{(a)}(t)$ is, for example, the output field of a semiconductor laser], (7), (9), and (10) lead to the interesting result

$$
\begin{aligned}
\operatorname{RIN}\left(\Omega, z, \beta_{\mathrm{FM}}, \Omega_{1}\right)= & {\left[\sum_{k=-\infty}^{\infty} J_{k}^{2}\left(2 \beta_{\mathrm{FM}} \sin \frac{\beta_{0}^{\prime \prime} z \Omega_{1}}{2}\right)\right.} \\
& \left.\cdot \operatorname{RIN}^{(a)}\left(\Omega-k \Omega_{1}, \frac{z \Omega}{\Omega-k \Omega_{1}}\right)\right] \\
+ & \operatorname{RIN}^{(b)}(\Omega, z)
\end{aligned}
$$

where $\operatorname{RIN}^{(b)}(\Omega, z)$ is given by (12) and $\operatorname{RIN}^{(a)}(\Omega, z)$ is the RIN of the source after propagation when there is no modulation. Equation (13) is obtained by using (9)-(11) in (7), interchanging the order of integration and summation, and making the temporary change of variables $\Omega \rightarrow \Omega^{\prime}+k \Omega_{1}$ and $z \Omega \rightarrow$ $z^{\prime} \Omega^{\prime}$ in order to evaluate the resulting integral. The right-hand side of (13) reduces to $\operatorname{RIN}^{(a)}(\Omega, z)$ for the case $\beta_{\mathrm{FM}}=0$ and to $\operatorname{RIN}^{(b)}(\Omega, z)$ for the pure carrier case $\operatorname{RIN}^{(a)}(\Omega, z)=0$.

Equation (13) was verified experimentally using the setup in Fig. 1 containing a 1550-nm DFB semiconductor laser coupled to $\mathrm{SMF}$ through an optical isolator, an external $\mathrm{LiNbO}_{3}$ phase modulator driven at power levels up to about $30 \mathrm{dBm}, 50 \mathrm{~km}$ of SMF-28 single-mode telecommunications fiber, a 15-GHz PIN photodiode, a low-noise $0.1-27-\mathrm{GHz}$ microwave preamp, and a $50-\mathrm{GHz}$ spectrum analyzer. The setup was calibrated for RIN measurements by replacing the $50-\mathrm{km}$ fiber spool with an optical attenuator and measuring, for a range of attenuations, the photocurrent and microwave power in a narrow $(2 \mathrm{MHz})$ bandwidth at each desired measurement frequency (with no signal applied to the phase modulator). The procedure is automated in our setup and the results are fit to the known (quadratic) dependence of measured power on photocurrent in real time, yielding the in situ optical-to-electrical gain and the noise floor at each frequency.

Using the calibrated system, the RIN of the laser biased at $200 \mathrm{~mA}$ was measured for $\beta_{\mathrm{FM}}=0$ (i.e., no modulation) with 


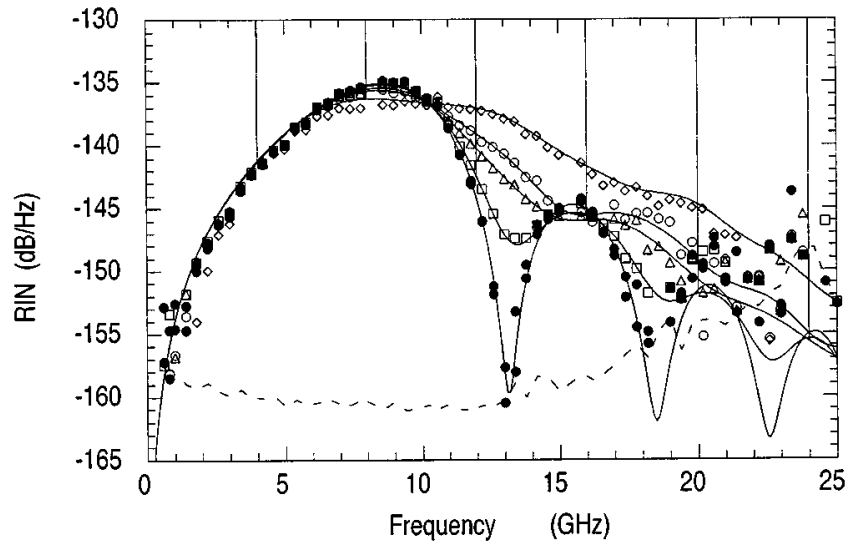

Fig. 2. Relative intensity spectrum (in $\mathrm{dB} / \mathrm{Hz}$ ) at $50 \mathrm{~km}$ for $4-\mathrm{GHz}$ phase modulation. The solid curves are from (13) with $\beta_{\mathrm{FM}}=0$ (dots), 0.34 (squares), 0.62 (triangles), 0.78 (circles), and 1.23 (diamonds); the vertical portions of these curves represent (delta function) signal components. The data gradually becomes noisy above $15 \mathrm{GHz}$ due to the effect of roll-off in the photodiode response. The lower, dashed line represents an estimate of the minimum value of RIN that could be measured meaningfully at the photocurrent available $(0.2 \mathrm{~mA}$ after $50 \mathrm{~km}$ propagation).

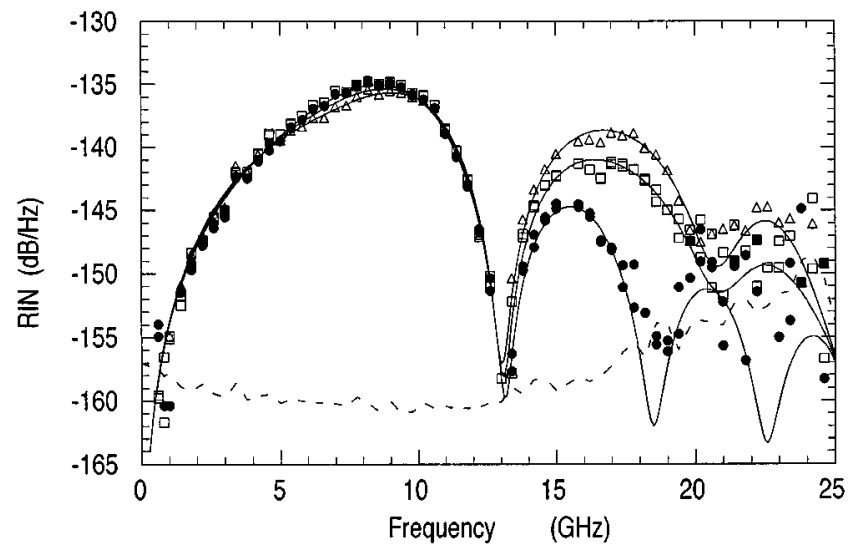

Fig. 3. Relative intensity spectrum (in $\mathrm{dB} / \mathrm{Hz}$ ) at $50 \mathrm{~km}$, for $12.8-\mathrm{GHz}$ phase modulation. The solid curves are from (13) with $\beta_{\mathrm{FM}}=0$ (dots), 0.35 (squares), 0.51 (triangles).

and without $50-\mathrm{km}$ propagation. These spectra were used as described in [11] to extract the following operating parameters for the laser and fiber: relaxation resonance angular frequency, $\Omega_{0}=2 \pi \times 11.5 \mathrm{GHz}$; relaxation damping rate, $\gamma_{0}=8.9 / \mathrm{ns}$; linewidth enhancement factor, $\alpha=-5.6$; laser linewidth, $\Delta \nu=0.95 \mathrm{MHz}$; photon lifetime $\tau_{p h}=5 \mathrm{ps}$, and fiber dispersion, $\beta_{0}^{\prime \prime}=-19.5 \mathrm{ps}^{2} / \mathrm{km}$ (i.e., $D=15.7 \mathrm{ps} / \mathrm{nm} / \mathrm{km}$ ). The resulting model ([11] with these parameters) was designated as the function $\operatorname{RIN}^{(a)}(\Omega, z)$.

Next, an $\Omega_{1}=2 \pi \times 4 \mathrm{GHz}$ sine wave was applied to the input of the phase modulator and the RIN was again measured before and after $50-\mathrm{km}$ propagation. Measurement of the RIN with no fiber confirmed that it was unaffected by the phase modulator alone. The RIN with fiber was measured at each of several different modulation levels (i.e., values of $\beta_{\mathrm{FM}}$ ) and the results are shown in Fig. 2. The data for $\beta_{\mathrm{FM}}>0$ was fit to (13) [restricting the sum to $|k| \leq 3$ and using $\operatorname{RIN}^{(a)}(\Omega, z)$ described above] yielding the values $\beta_{\mathrm{FM}}=0.34,0.62,0.78$, and 1.23 .

The presence of large-signal intensity variations in the receiver is a limiting factor in the accuracy of the above measurements. A large signal saturates (thus, decalibrates) the gain of the microwave preamp and may also lead to postdetection harmonic mixing in the preamp and/or in the spectrum analyzer front-end. In order to eliminate the possibility of such errors, and to emphasize the fact that the presence of intensity modulation at the receiver is not required for an observable affect on the RIN, a second set of measurements was performed using phase modulation at $\Omega_{1}=2 \pi \times 12.8 \mathrm{GHz}$. For this modulation frequency there is essentially no intensity variation due to modulation at either the transmitter or at the receiver, because $12.8 \mathrm{GHz}$ and its multiples are at nulls in the phase-to-amplitude conversion response for $z_{1}=50 \mathrm{~km}$ of fiber [that is, because $-(1 / 2) \beta_{0}^{\prime \prime} z_{1} \Omega_{1}^{2}=\pi$ at this modulation frequency]. Fitting to (13) in this case (see Fig. 3) yields values of $\beta_{\mathrm{FM}}=0.35$ and 0.51 and improved fits compared to the results at $4 \mathrm{GHz}$. Only relatively low levels of phase modulation could be obtained at this frequency due to roll-off in the phase modulator response, but a significant effect on the RIN, in agreement with theory, was still observed.

\section{REFERENCES}

[1] E. Bochove, E. M. de Carvalho, and J. E. R. Filho, "FM-AM conversion by material dispersion in an optical fiber," Opt. Lett., vol. 6, no. 2, pp. $58-60,1981$.

[2] G. J. Meslener, "Chromatic dispersion induced distortion of modulated monochromatic light employing direct detection," IEEE J. Quantum Electron., vol. QE-20, pp. 1208-1216, Oct. 1984.

[3] A. Chraplyvy, R. Tkach, L. Buhl, and R. Alferness, "Phase modulation to amplitude modulation conversion of CW laser light in optical fibers," Electron. Lett., vol. 22, no. 8, pp. 409-411, 1986.

[4] K. Petermann, "FM-AM noise conversion in dispersive single-mode fiber transmission lines," Electron. Lett., vol. 26, no. 25, pp. 2097-2098, 1990.

[5] S. Yamamoto, N. Edagawa, H. Taga, Y. Yoshida, and H. Wakabayashi, "Analysis of laser phase noise to intensity noise conversion by chromatic dispersion in intensity modulation and direct-detection opticalfiber transmission," J. Lightwave Technol., vol. LT-8, pp. 1716-1722, Nov. 1990.

[6] M. R. Phillips, T. E. Darcie, D. Marcuse, G. E. Bodeep, and N. J. Frigo, "Nonlinear distortion generated by dispersive transmission of chirped intensity-modulated signals," IEEE Photon. Technol. Lett., vol. 3, no. 5, pp. 481-483, 1991.

[7] J. Wang and K. Petermann, "Small signal analysis for dispersive optical fiber communication systems," J. Lightwave Technol., vol. 3, no. 5, pp. 96-100, 1992.

[8] K. Petermann and J. Wang, "Large signal analysis of FM-AM conversion in dispersive optical fibers and its application to PCM systems," Electron. Lett., vol. 27, no. 25, pp. 2347-2348, 1991.

[9] Y. Gao and M. Leich, "Nonlinear distortion and intensity noise induced by laser frequency chirping and fiber dispersion in multichannel intensity modulated systems," Eur. Trans. Telecom, vol. 4, no. 4, pp. 471-478, 1993.

[10] W. K. Marshall, B. Crosignani, and A. Yariv, "Laser phase noise to intensity noise conversion by lowest-order group velocity dispersion in optical fiber: Exact theory," Optics Lett., to be published.

[11] W. K. Marshall, J. Paslaski, and A. Yariv, "Reduction of the relative intensity noise of the output field of semiconductor lasers due to propagation in dispersive optical fiber," Appl. Phys. Lett., vol. 68, no. 18, pp. 2496-2498, 1996. 